\title{
Experimental approaches to low $x$ at HERA
}

\author{
Jörg Gayler \\ DESY, Notkestrasse 85, 22603 Hamburg, Germany \\ E-mail: gayler@mail.desy.de
}

\begin{abstract}
Data are presented on the production of jets and $\pi^{0}$ mesons at low Bjorken $x$ in a kinematic region where standard DGLAP evolution in $Q^{2}$ gives little phase space for high $p_{t}$ particle and jet production. The data are compared with various QCD models based on different treatments of parton emissions at small $x$.
\end{abstract}

\section{Introduction}

Experimentally QCD dynamics at low $x$ are mostly studied in nucleon structure functions (for recent results see [1, 2]), heavy quark production in deep inelastic scattering (DIS) [3], and forward jets and particle production (see also [4]). Results on the latter are presented here困. "Forward" refers in the present context of $e p$ interactions to the region close to the outgoing proton beam. This region is particularly interesting, as the large energies available at small $x$ give rise to a large phase space for gluon ladders which are sensitive to different QCD evolution schemes as indicated in Fig. 1. Due to
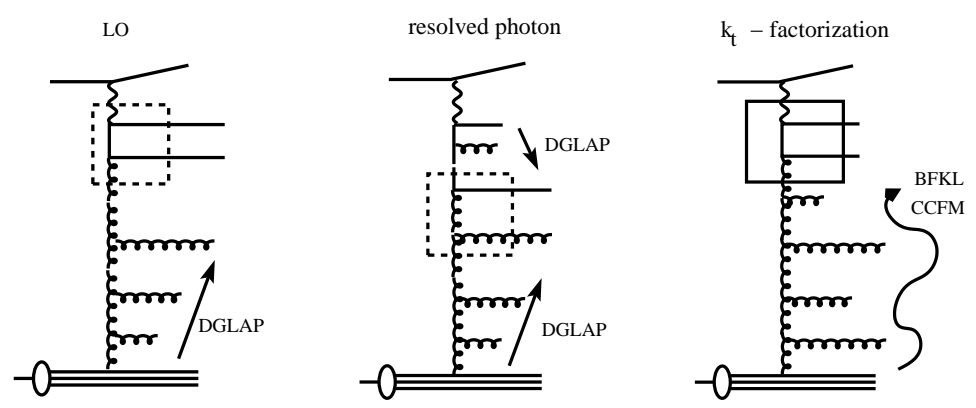

Figure 1. Different evolution schemes. Left: Direct photon coupling to leading order matrix element of the hard process. The arrow indicates the ordering in increasing $k_{t}$ in DGLAP evolution. Center: same, but for resolved photons. Right: BFKL and CCFM approach with ordering in energy and angle respectively.

the increasing virtualities $k_{t}$ towards the hard interaction in the DGLAP [5] evolution scheme (Fig. 1, left), large energy jets and hadrons with substantial $p_{t}$ are suppressed in

$\ddagger$ Presented at XXXII International Symposium on Multiparticle Dynamics, Alushta, Crimea, 7. - 13th September 2002 
the forward direction. If a resolved hadronic photon structure is considered, this strict ordering is broken (Fig. 1, centre). Finally, based on the BFKL [6] (CCFM) [7] equation, ordering in energy (angle) is obtained with $k_{t}$ factorisation. In this case substantial $p_{t}$ can be expected anywhere in the ladder (Fig. 1, right).

NLO pQCD calculations to order $\alpha_{s}^{2}$ are not able to describe forward jet data at small $Q^{2}$ where the NLO corrections are very large [8].

In this report data [9] on forward jet and $\pi^{0}$ production (see ref. [10] for previous data) are compared with several QCD based Monte Carlo (MC) models. The RAPGAP MC model [11] combines leading order (LO) matrix elements with DGLAP parton showers and is used with and without resolved virtual photon contributions. The treatment of higher orders in the ARIADNE program [12], which is based on the Colour Dipole Model (CDM) [13], leads to unordered parton emissions, as expected in the BFKL approach. Finally, CASCADE [14] corresponds to a solution of the CCFM equation.

\section{Results}

Jets are selected using the inclusive $k_{t}$ algorithm in the kinematic range $5<Q^{2}<75$ $\mathrm{GeV}^{2}, 7<\theta_{\text {jet }}<20^{\circ}$. Substantial jet $p_{t}$ and jet energy is required by the conditions $0.5<p_{t j e t}^{2} / Q^{2}<2$ and $x_{j e t}=E_{\text {jet }} / E_{p}>0.035$, where $E_{p}$ is the incident proton energy. The $\pi^{0}$ mesons are selected in a similar kinematic range with transverse momentum in the hadronic centre of mass system (CMS) $p_{t, \pi}^{*}>2.5$ or $>3.5 \mathrm{GeV}$. The results in Fig. 2 show that in both cases the DGLAP RAPGAP model with only direct photon
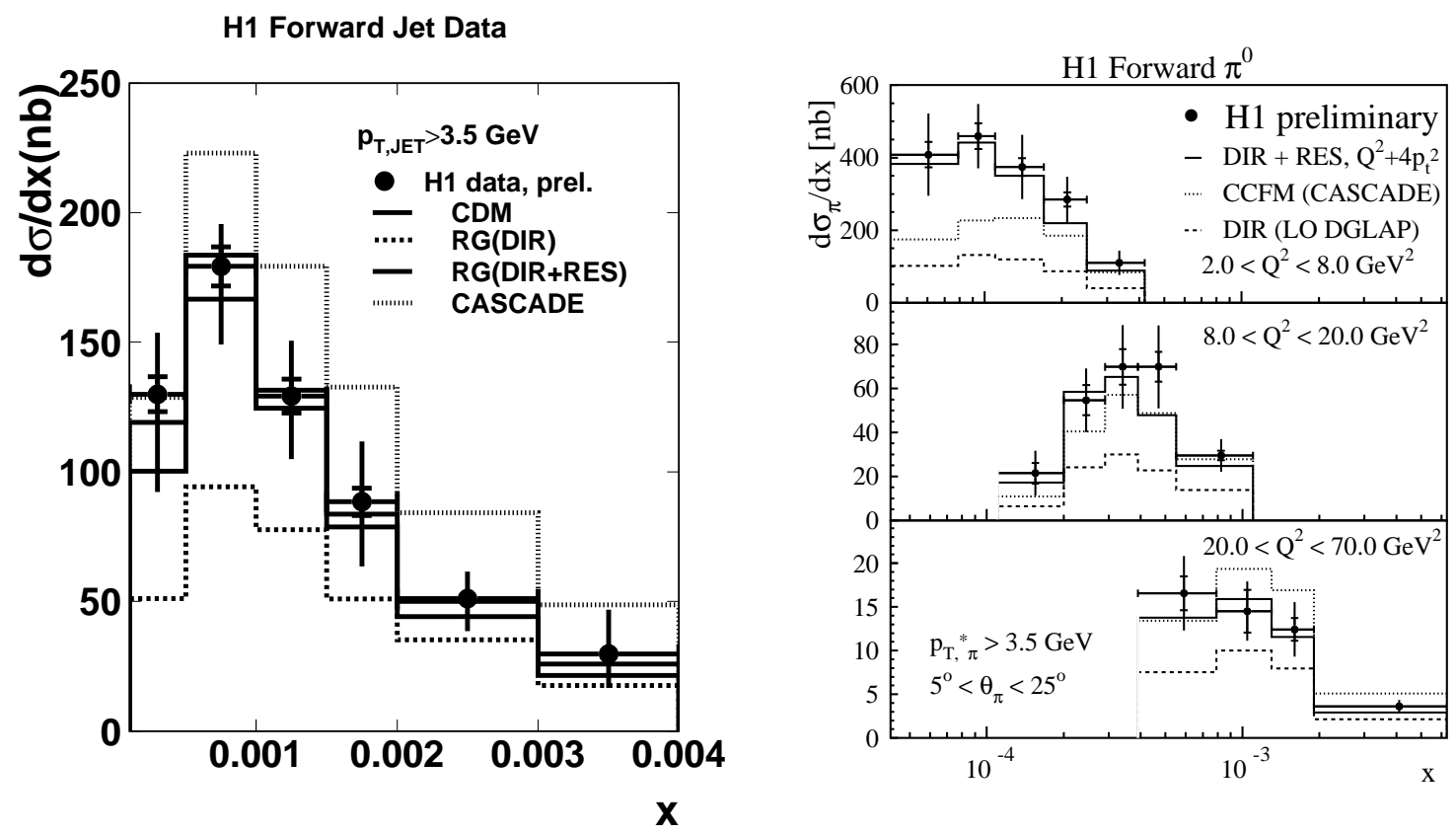

Figure 2. Forward jet (left) and forward $\pi^{0}$ (right) data with predictions of RAPGAP with direct and resolved $\gamma$ interactions and DGLAP LO parton showers, ARIADNE based on the Colour Dipole Model (CDM) and CASCADE based on the CCFM equation. 
interactions is well below the data. However, a very good description is achieved if resolved photon interactions are included. Also the CDM model describes the jet data very well. The CCFM CASCADE model predicts cross sections that are too large at large $x(x \gtrsim 0.001)$.

It is interesting to see (Fig. 3), that the transverse energy flow between the photon coupling and the $\pi^{0}$ meson also does not follow the expectations from direct photon interactions with DGLAP parton showers, which predict a larger amount of $E_{t}$ close to the virtual photon than is observed in the data.

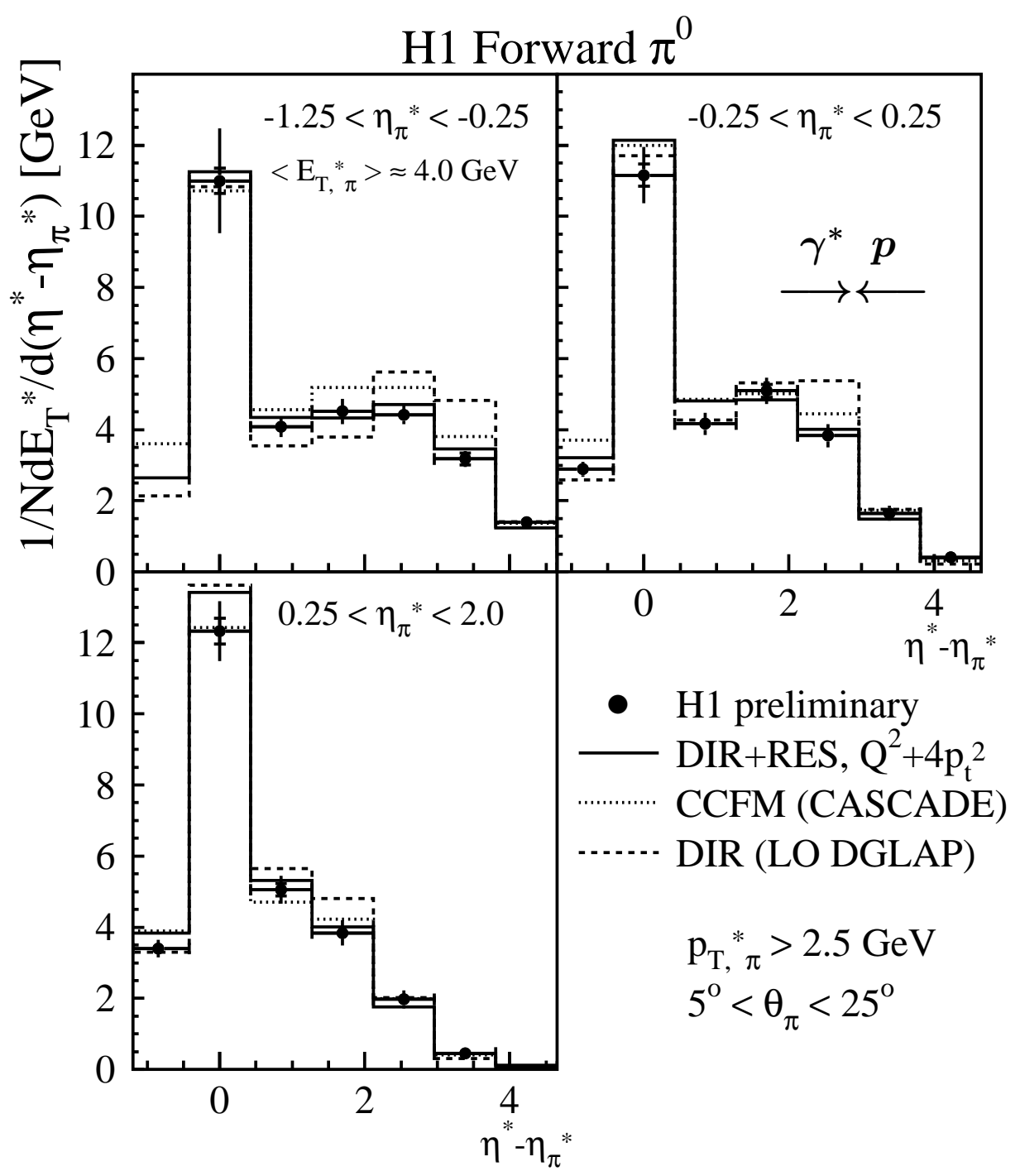

Figure 3. Transverse energy flow relative to the $\pi^{0}$ for different ranges of pseudo rapidity $\eta_{\pi^{*}}$ in the hadronic CMS compared with the predictions of CASCADE, and RAPGAP with DGLAP parton showers with (DIR+RES) and without (DIR) resolved virtual photon interactions. The contribution from the $\pi^{0}$ itself is included in the energy flow. 


\section{Conclusion}

The presented DIS forward jet and $\pi^{0}$ data can be described by LO matrix elements and DGLAP parton showers only if resolved photon interactions are included. The CDM model, which contains photon emissions unordered in $k_{t}$, describes the jet data very well. The CASCADE MC does not describe the $x$ dependence of the data.

\section{Acknowledgments}

I am grateful to Hannes Jung, Lidia Goerlich, Martin Karlsson, Paul Newman, Grazyna Nowak and Jacek Turnau for discussions or comments. I thank the organisers for an interesting conference, in particular Elena Kolganova for kind support.

\section{References}

[1] A. Kappes [ZEUS Collaboration], "Structure function results from ZEUS", hep-ex/0210032, proceedings ICHEP 2002, Amsterdam;

Z. Zhang [H1 Collaboration] "Structure function results from H1", ibid.

[2] J. Gayler, "Proton Structure Functions Measurements from HERA", these proceedings, hep-ex/0211051,

[3] N. Zotov, "Heavy quark production with BFKL and CCFM Dynamics", these proceedings; S. P. Baranov, H. Jung, L. Jonsson, S. Padhi and N. P. Zotov, Eur. Phys. J. C 24 (2002) 425.

[4] C. Merino, "Correlation between average pt and jet multiplicities from the BFKL pomeron", these proceedings.

[5] Y. L. Dokshitzer, Sov. Phys. JETP 46 (1977) 641;

V. N. Gribov and L. N. Lipatov, Yad. Fiz. 15 (1972) 1218; Yad. Fiz. 15 (1972) 781;

G. Altarelli and G. Parisi, Nucl. Phys. B 126 (1977) 298.

[6] E. A. Kuraev, L. N. Lipatov and V. S. Fadin, Sov. Phys. JETP 44 (1976) 443; Sov. Phys. JETP 45 (1977) 199;

I. I. Balitsky and L. N. Lipatov, Sov. J. Nucl. Phys. 28 (1978) 822.

[7] M. Ciafaloni, Nucl. Phys. B 296 (1988) 49;

S. Catani, F. Fiorani and G. Marchesini, Phys. Lett. B 234 (1990) 339, Nucl. Phys. B 336(1990)18;

G. Marchesini, Nucl. Phys. B 445 (1995) 49.

[8] C. Adloff et al. [H1 Collaboration], Phys. Lett. B 542 (2002) 193.

[9] H1 Collab., contributions to ICHEP 2002, Abstracts 1000 and 1001.

[10] C. Adloff et al. [H1 Collab.], Nucl. Phys. B 538 (1999) 3; Phys. Lett. B 462 (1999) 440;

J. Breitweg et al. [ZEUS Collab.], Phys. Lett. B 474 (2000) 223.

[11] H. Jung, Comput. Phys. Commun. 86 (1995) 147.

[12] L. Lonnblad, Comput. Phys. Commun. 71 (1992) 15.

[13] B. Andersson, G. Gustafson and L. Lonnblad, Nucl. Phys. B 339 (1990) 393.

[14] H. Jung, arXiv:hep-ph/9908497. 